\title{
ТАКТИКА ХИРУРГИЧЕСКОГО ЛЕЧЕНИЯ БОЛЬНЫХ ПО ПОВОДУ МНОЖЕСТВЕННЫХ ГРЫЖ МЕЖПОЗВОНКОВЫХ ДИСКОВ ШЕЙНОГО ОТДЕЛА ПОЗВОНОЧНИКА
}

\author{
Е. Г. Педаченко, М. В. Хижняк, Ю. Е. Педаченко, А. Ф. Танасийчук, \\ В. А. Крамаренко, А. Н. Фурман
}

Институт нейрохирургии имени А. П. Ромоданова НАМН Украины, г. Киев

\section{TACTICS OF SURGICAL TREATMENT OF THE PATIENTS, SUFFERING MULTIPLE HERNIAS OF INTERVERTEBRAL DISCS OF CERVICAL VERTEBRAL COLUMN}

\author{
E. G. Pedachenko, M. V. Khyzhnyak, Yu. E. Pedachenko, A. F. Tanasiychuk, \\ V. A. Kramarenko, A. N. Furman \\ Romodanov Institute of Neurosurgery, Kyiv
}

\begin{abstract}
Реферат
Цель исследования. Улучшение результатов хирургического лечения больных по поводу множественных грыж межпозвонковых дисков (МПД) шейного отдела позвоночника.

Материалы и методы. Проанализированы результаты хирургического лечения 65 пациентов, в том числе 27 (41,5\%) женщин, $38(58,5 \%)$ мужчин, в возрасте в среднем $(50 \pm 0,56)$ года, продолжительность заболевания в основном $1-3$ года.

Результаты. Все пациенты активизированы в ближайшие 24 ч после оперативного вмешательства. Осложнений, рецидива болевого синдрома, прогрессирования неврологических симптомов после операции не отмечали.

Обсуждение. Полученные положительные результаты свидетельствуют об эфрфективности примененных методов хирургического лечения.

Выводы. 1. Стандартная микродискэктомия с применением переднего доступа в сочетании с пункционной лазерной микродискэктомией (ПЛМ) либо фенестрацией, выполненная у больных по поводу множественных грыж МПД шейного отдела позвоночника, позволяет:

- устранить дискогенную компрессию;

- минимизировать хирургическую травму;

- уменьшить продолжительность реабилитации после операции.

2. Сочетанное применение методов хирургического лечения позволило предупредить дальнейшее грыжеобразование на измененном смежном уровне позвоночно-двигательного сегмента.

Ключевые слова: шейный отдел позвоночника; множественные грыжи межпозвонковых дисков; дискэктомия.

Abstract

Objective. Improvement of the surgical treatment results in patients, suffering multiple hernias of intervertebral discs of cervical vertebral column.

Materials and methods. Results of surgical treatment of 65 patients were analyzed, including 27 (41.5\%) women, 38 (58.5\%) men, ageing $(50 \pm 0.56)$ yrs at average, and the disease duration $1-3$ yrs mainly.

Results. All the patients were activated in immediate $24 \mathrm{~h}$ after operative intervention. Complications, the pain syndrome recurrence, progression of neurological symptoms were not noted postoperatively.

Discussion. The positive results obtained witnesses efficacy of the applied methods of surgical treatment.

Conclusion. 1. Standard microdiscectomy, using anterior access in combination with a puncture laser microdiscectomy or fenestration,

performed in the patients, suffering multiple hernias of intervertebral discs of cervical vertebral column, permits:

- to eliminate a discogenic compression;

- to minimize surgical trauma;

- to reduce the postoperative rehabilitation duration.

2. Combination of the surgical treatment methods have permitted to warnfurther development of hernias in adjacent level of vertebromotor segment.

Keywords: cervical vertebral column; multiple hernias of intervertebral discs; discectomy.
\end{abstract}

По данным литературы, заболевания позвоночника занимают пятое место в структуре причин госпитализации пациентов и третье место - в структуре хирургических вмешательств, наиболее частыми причинами являются дегенеративнодистрофические заболевания, травматическое повреждение шейного отдела позвоночника, а также гене- тические факторы $[1,2]$. Формирование дискогенных нейрокомпрессионных синдромов, обусловливающих нередко инвалидизацию больных, определяет социальную значимость проблемы [3].

Поражение смежных мПД сопровождается формированием клинически значимой грыжи больших размеров - на одном уровне и протрузии - в смежном позвоночно-двигательном сегменте.

Дополнительное применение ПЛМ и микрофенестрации на смежном уровне во время выполнения стандартной микродискэктомии не влияет на тяжесть хирургической травмы.

Цель исследования: улучшение результатов хирургического лече- 
ния больных по поводу множественных грыж МПд шейного отдела позвоночника.

\section{МАТЕРИАЛЫ И МЕТОДЫ ИССЛЕДОВАНИЯ}

Проанализированы результаты хирургического лечения 65 пациентов по поводу множественных грыж МПд шейного отдела позвоночника в отделении малоинвазивной и лазерной спинальной нейрохирургии в период 1997 - 2016 гг., в том числе 27 (41,5\%) женщин и 38 (58,5\%) мужчин.

Возраст оперированных больных в среднем $(50 \pm 0,56)$ года, большинство из них - среднего возраста. Продолжительность заболевания преимущественно от 1 до 3 лет. При обосновании показаний к оперативному вмешательству учитывали возраст пациентов, анамнез заболевания, выраженность клинических и неврологических симптомов, результаты инструментальных методов исследования.

У 9 пациентов продолжительность заболевания составляла 2 - 6 мес, у $34-6-24$ мес, у $15-24-60$ мес, у 4 - 60 - 120 мес. Поражение на уровне $\mathrm{C}_{\mathrm{III}}-\mathrm{C}_{\mathrm{IV}}, \mathrm{C}_{\mathrm{IV}}-\mathrm{C}_{\mathrm{V}}$ отмечено у 3 пациентов, $\mathrm{C}_{\mathrm{IV}}-\mathrm{C}_{\mathrm{v}}, \mathrm{C}_{\mathrm{V}}-\mathrm{C}_{\mathrm{VI}}-\mathrm{y} 17, \mathrm{C}_{\mathrm{V}}$ $-\mathrm{C}_{\mathrm{VI}}, \mathrm{C}_{\mathrm{VI}}-\mathrm{C}_{\mathrm{VII}}-\mathrm{y} 41, \mathrm{C}_{\mathrm{VI}}-\mathrm{C}_{\mathrm{VI}} \mathrm{I}, \mathrm{C}_{\mathrm{VII}}-\mathrm{T}_{\mathrm{I}}$ $-\mathrm{y} 4$.

Радикулопатия отмечена у 42 больных, миелопатия-у 5, миелорадикулопатия-у 18.

Таблица 1. Динамика регресса болевого синдрома по шкале ВАШ в группах пациентов

\begin{tabular}{|c|c|c|}
\hline \multirow{2}{*}{ Сроки наблюдения } & \multicolumn{2}{|c|}{ Величина показателя, баллов в группах ( $\bar{x} \pm$ m) } \\
\hline & $1-\breve{~}$ & $2-\breve{~}$ \\
\hline До операции & $9,4 \pm 1,6$ & $9,6 \pm 1,2$ \\
\hline \multicolumn{3}{|l|}{ После операции } \\
\hline $24-484$ & $1,5 \pm 1,4$ & $1,3 \pm 1,1$ \\
\hline $6 \mathrm{mec}$ & $1,2 \pm 1,3$ & $1,3 \pm 1,1$ \\
\hline $12 \mathrm{мес}$ & $0,7 \pm 0,23$ & $1,3 \pm 1,1$ \\
\hline
\end{tabular}

Таблица 2. Динамика регресса болевого синдрома по шкале NPS в группах пациентов

\begin{tabular}{|c|c|c|}
\hline \multirow{2}{*}{ Сроки наблюдения } & \multicolumn{2}{|c|}{ Величина показателя, баллов в группах $(\bar{x} \pm m)$} \\
\hline & 1-й & $2-\breve{n}$ \\
\hline До операции & $7,88 \pm 1,6$ & $7,65 \pm 1,4$ \\
\hline \multicolumn{3}{|l|}{ После операции } \\
\hline $24-484$ & $1,58 \pm 1,2$ & $1,55 \pm 1,2$ \\
\hline $6 \mathrm{mec}$ & $1,38 \pm 1,3$ & $1,55 \pm 1,2$ \\
\hline $12 \mathrm{mec}$ & $1,22 \pm 1,23$ & $1,55 \pm 1,2$ \\
\hline
\end{tabular}

В целях уточнения диагноза всем пациентам, кроме клинико-неврологического обследования, проведены спондилография в стандартных проекциях и с функциональными пробами, магниторезонансная томография (МРТ), компьютерная томография (у 36), электронейромиография (у 16). Всем пациентам перед оперативным вмешательством проведен курс консервативной терапии.

В зависимости от выбранной хирургической тактики больные распределены на 2 группы.

У 34 (52,3\%) больных (1-я группа) выполнена стандартная микродискэктомия с применением переднего доступа и последующей установкой кейджа на клинически значимом уровне, дополнительно - Плм на смежном уровне при наличии (по данным МРТ) протрузии МПд.

У 31 (47,7\%) больного (2-я группа) осуществлена стандартная микродискэктомия с использованием переднего доступа и последующей установкой кейджа на клинически значимом уровне, дополнительно фенестрация МПД на смежном уровне при наличии (по данным МРТ) его протрузии.

Показаниями к проведению ПЛМ была протрузия (гидрофильной плотности, по данным МРТ) МПД на смежном уровне;

микрофенестрации - протрузия (дегидратированная, по данным MPТ) МПД на смежном уровне.

\section{РЕЗУЛЬТАТЫ}

Все пациенты активизированы в ближайшие 24 ч после оперативного вмешательства. Осложнений не было. Продолжительность госпитализации до 3 дней. Оценка результатов хирургического лечения представлена в табл. 1, 2.

Рецидив болевого синдрома, прогрессирование неврологических симптомов после операции в обеих группах не отмечены.

По данным контрольной МРТ в динамике дальнейшее грыжеобразование на смежном уровне позвоночно-двигательного сегмента не обнаружено.

\section{ОБСУЖДЕНИЕ}

Метод передней дискэктомии с последующим спондилодезом впервые внедрили G. W. Smith и R. А Robinson, R. W. Bailey [4 - 7], сегодня он является высокоэффективным методом хирургического лечения пациентов по поводу грыжи МПД шейного отдела позвоночника.

Снижение внутридискового давления путем фенестрации передних отделов фиброзного кольца у 30 пациентов впервые описал L. Hult в 1951 г.

Метод ПЛМ, суть которого заключается в снижении внутридискового давления без радикального удаления студенистого ядра, предложен в 1986 г. D. S. Chоу и соавторами [8].

В настоящее время данные, обобщающие результаты лечения неврологических проявлений многоуровневых грыж МПД шейного отдела позвоночника путем применения комбинированных хирургических методов, немногочисленны. Полученные в нашем исследовании положительные результаты в ближайшем и отдаленном периоде свидетельствуют об эффективности сочетанного применения методов хирургического лечения больных.

\section{выводы}

1. Стандартная микродискэктомия с применением переднего доступа в сочетании с ПЛМ либо фенестрацией у больных, выполненная по поводу множественных грыж МПД шейного отдела позвоночника, позволяет: 
- устранить дискогенную компрессию;

- минимизировать хирургическую травму;
- уменьшить продолжительность послеоперационной реабилитации пациентов.

2. Сочетанное применение методов хирургического лечения по- зволило предупредить дальнейшее грыжеобразование на измененном смежном уровне позвоночно-двигательного сегмента.

\section{REFERENCES}

1. Antonov IP. Patogenez i diagnostika osteohondroza pozvonochnika i ego nevrologicheskih projavlenij: sostojanie problemy i perspektivy izuchenija. Zhurn nevropatologii i psihiatrii. 1986;86(4):481-48. [In Russian].

2. Iyer S, Kim HJ. Cervical radiculopathy. Curr Rev Musculoskelet Med. 2016;9(3):272-80. doi:10.1007/s12178-016-9349-4. PMID:27250042.

3. Vereshhagin NV, Bragina LK, Blagoveshhenskaja NS, i dr. Spravochnik po nevrologii. 3-e izdanie. Moskva: Medicina; 1989. [In Russian].

4. Smith GW, Robinson RA. The treatment of certain cervical spine disorders by anterior removal of the intervertebral disc and interbody fusion. J Bone Joint Surg. 1958;40:607-24. Available from: https:// www.ncbi.nlm.nih.gov/pmc/articles/PMC5400196/

5. Bailey RW, Badgley CE. Stabilization of the cervical spine by anterior fusion. J Bone Joint Surg [Am]. 1960;42(A):565-94. PMID:13848906

6. Dohrmann GJ, Hsieh JC. Long-term results of anterior versus posterior operations for herniated cervical discs: analysis of 6,000 patients. Med Princ Pract. 2014;23(1):70-3. Available from: www.karger.com/Article/FullText/351887

7. Daglı M, Er U, Simşek S, Bavbek M. Late results of anterior cervical discectomy and fusion with interbody cages. Asian Spine J. 2013;7(1):34-8. doi:10.4184/asj.2013.7.1.34.PMID:23508467.

8. Choy DS, Ngeas S. Percutaneous laser disc decompression in spinal stenosis. J Clin Laser Med Surg. 1998. Apr: 16(2):123-5. 